\title{
Detection of Plasmodium species and parasite density among patients attending some selected hospitals in Sokoto Metropolis
}

\author{
Bello Muntari * and Bello Abubakar Tureta \\ Department of Medical Microbiology, School of Medical Laboratory Science, Usmanu Danfodiyo University, P.M.B. 2346, \\ Sokoto, Nigeria.
}

Publication history: Received on 17 October 2019; revised on 19 November 2019; accepted on 30 November 2019

Article DOI: https://doi.org/10.30574/gscbps.2019.9.3.0196

\begin{abstract}
Malaria remains a major cause of mortality in developing countries; it is endemic throughout Nigeria with seasonal variation in different geographic zones of the country. More than 120 Plasmodium Species are known, but only six infect humans: P. falciparum, P. vivax P. ovale; Curtisi and willikeri, P. malariae, P. knowlesi. The aim of this study was to detect the Plasmodium species and parasite density among patient attending some selected hospitals in Sokoto metropolis. A total of 297 samples were collected and analyzed using thick and thin blood films. An overall prevalence of $28 \%$ was obtained and Plasmodium falciparum was responsible for all the cases of infection. Based on gender, prevalence rates of $57 \%$ and $43 \%$ were obtained for male and female patients respectively. The mean parasite density of $690 \mu / 1$ and $450 \mu / \mathrm{l}$ were obtained for male and female subjects respectively. Based on age groups, the prevalence rates of $43 \%$, $29 \%, 17 \%, 6 \%$ and 5\% were recorded for $\leq 14$ years, $15-24$ years, $25-34$ year, 35-44 years and $45-54$ years respectively. Statistical analysis using Pearson's correlation showed a significant $r$ value of 0.020 while $p$ value was insignificant (0.731). Based on gender, a significant correlation coefficient value of 0.103 was obtained and the p value (0.76) was insignificant. This study revealed that parasite density decreases with increase in age. Females had higher parasite density than males and Plasmodium falciparum was the only specie identified. Parasite density estimation using assumed WBC count in the diagnosis of malaria is recommended.
\end{abstract}

Keywords: Detection; Plasmodium species; Parasite density; Sokoto metropolis

\section{Introduction}

Malaria is a vector-borne parasitic tropical disease found in 91 countries worldwide [1]. More than 120 Plasmodium species are known, but only six infect humans; P.falciparum, P.vivax P.Ovale;Curtisi and willikeri, P.malariae, P.knowlesi. It has been stated that this disease is eradicated from temperate countries steadily over the last 100 years [2]. The disease is transmitted by the bite of the female Anopheles mosquito. Disease occurrence depends on environmental suitability for local vectors in terms of altitude, climate, vegetation, and implementation of control measures, and hence is it linked with poverty, natural disasters, and war [3]. Less common transmission routes are from mother to child, or via blood transfusion. It has been estimated that half of the world population ( 3.5 billion people) lived in malaria transmission area in 2010, the profound effect of malaria is common in sub Saharan Africa countries, and most estimated 1 million death caused annually by malaria occur in Africa which can affect an average African child up to 13 times there by making it a leading cause of under-five mortality, which include pregnant women and their unborn children killing an African child in every 30 seconds [1].

Plasmodium falciparum; produces high levels of blood-stage parasites that sequester in critical organs in all age groups and cause severe anemia in African children, it causes majority of deaths [4]. Plasmodium falciparum has a worldwide

\footnotetext{
${ }^{*}$ Corresponding author

E-mail address: bmukhtar18@yahoo.com
} 
distribution but predominantly in Africa. An estimated one million people are killed by this plasmodium falciparum every year. Plasmodium falciparum invades young and mature erythrocytes, the infected cell do not become enlarged and distorted, drug resistance infection of Plasmodium falciparum is the commonest challenge in many part of the world although all the six species of Plasmodium infecting humans have been recorded. P.falciparum may be treated with the use of mefloquine, quinine and pyrimethamine-sulfadoxine [2].

Plasmodium vivax; usually produces milder disease, but can be severe, and recurrent which bring significant associated morbidity. It possess a greatest threat than other species but have a wider distribution and predominate in many countries outside Africa [5]. Vivax can only invade young immature red blood cells, and the infected cells are usually enlarged. This strain has a dormant liver stage that can activates and invades the blood cell after a month, or years, therebycausing relapse. Chloroquine is a drug of choice for suppression and therapeutic treatment of P.vivax followed by primaquine for radical cure.

Plasmodium ovale; has two (2) species, these are: Plasmodium ovalecurtisi and Plasmodium ovalewallikeri which are understudied [6], but the severity of the illness is generally similar to uncomplicated vivax malaria. Endemic mainly in west Africa but it can occur in Asia and south America. It is biologically and morphologically very similar to P.vivax, but differs from P.vivax because it affect individual with negative duffy blood group, which is the case with many resident in sub Saharan Africa. This explains a greater prevalence of P. ovale in Africa [7]. It invades only young pliable erythrocytes and the host cell become enlarged and distorted usually in an oval shaped. Treatment includes the use of primaquine to prevent relapse as in P.vivax [8].

Plasmodium malariae; Also found worldwide but primarily in sub-tropical and temperate regions, but less prevalence. Plasmodium malariae can invades only mature erythrocytes with relatively rigid cell membrane; as a result, the parasites growth must conform to the size and shape of the red blood cells [9].

Plasmodium Knowlesi; primarily a zoonotic infection encountered in South East Asia that can cause severe malaria [10]. It resembles Plasmodium malariae in morphology but parasite are reported to be high than in P. malariae and clinical symptoms are more severe and life threatening [11].

The life cycle of aforementioned Plasmodium species occurs when mosquito causes an infection by bite. First, the sporozoites enter the blood stream and migrates to the liver where they infect liver cells, and multiply into merozoites. The merozoites will rupture the liver cells and return to the blood stream, where they infect the red blood cells and developed in to ring form called trophozoite and schizonts, which in turn produce further merozoites. Sexual form are produced by the merozoites (gametocytes), which if taken up by a mosquito, will infect the mosquito and continue the life cycle [4].

Malaria is divided into two with regard to clinical disease presentations: which include uncomplicated and severe malaria [1]. Symptoms of uncomplicated malaria are non-specific, and can include fever, chills, body-aches, headache, cough, diarrhea, etc., making the clinical diagnosis unreliable in non-endemic areas [12]. Most common manifestations of severe malaria are convulsion, acute lung injury, which can progress to acute respiratory distress syndrome, acute kidney injury, typically presenting as acute tubular necrosis, and acidosis. Severe anemia is a common presentation in children, with frequent seizures [13].

The gold standard for malaria diagnosis remains light microscopy of stained blood films, thick films providing sensitivity and thin films allows speciation and quantitation, for accurate diagnosis. To find the density of parasite in thick blood film an assume white blood cell count of $8000 \mathrm{u} / \mathrm{l}$ provide a standard for quantification of prasitaemia, while species identification can be achieved by using thin film [14]. The main considerations when prescribing antimalarial drug are the infecting species because of the risk of drug resistance in order to prevent the burden of malaria in endemic areas [15].

Most prevention received by patients include the following: Pregnant women receive sulfadoxine-pyrimethamine at all antenatal care visits from second trimester (minimum dose interval of 1 month), Children aged 3-59 months isamodiaquine at treatment doses, Travelers take (atovaquone-proguanil, doxycycline, mefloquine as drug of choice for prophylaxis), HIV-infected patients receiving co-trimoxazole as prophylaxis for opportunistic infections are protected against malaria, Vaccination with RTS S/AS01 (Recombinant protein-based) in children (four doses for children aged 5-17 months) is the only registered malaria vaccine (still under evaluation), vector control or bite prevention, Long-lasting insecticide-treated bed nets most effective in high-transmission areas where vectors rest indoors at night, Indoor residual spraying with insecticides repellants [1]. 
The aim of this study is to detect Plasmodium species and estimate parasite density among patients suspected with malaria in some selected hospitals in sokoto metropolis. However, the specific objectives are:

- To determine the prevalence of malaria among patients attending specialist and Maryam Abacha women and children hospitals, Sokoto

- $\quad$ To detect the Plasmodium species circulating among patients diagnosed with malaria

- To estimate the parasite density among patients based on gender

- To determine prevalence of malaria among patients based on age

\section{Material and methods}

\subsection{Study design}

The study was cross sectional.

\subsection{Study area}

This study was conducted in two hospitals; Specialist Hospital and Maryam Abacha Women and Children hospital located in Sokoto metropolis, North-western Nigeria. The state is located between longitudes $11^{\circ} 30$ to $13^{\circ} 50$ East and latitude $4^{\circ}$ to $6^{\circ}$ North. It has a land area of about 28,232.37sq kilometer and stands at an altitude of $272 \mathrm{~m}$ above sea level near to the confluence of the Sokoto River and the Rima River. Sokoto state is at the extreme Northwest of Nigeria forming a border with Niger Republic. The state is in the dry Sahel surrounded by sandy terrain and Isolated hills with an average annual temperature of $28.3{ }^{\circ} \mathrm{C}\left(82.9^{\circ} \mathrm{F}\right)$. The weather is characterized by two seasons the wet and dry seasons. Rainfall (wet season) starts late around June and ends in September sometimes extending into October. The average annual rainfall is $550 \mathrm{~mm}$ with peak rainfall usually recorded in the month of August. The highest temperatures of $45^{\circ} \mathrm{C}$ during the hot season are experienced in the months of March and April. Harmattan, a dry cold and dusty condition is experienced between the months of November and February. Sokoto State had a population of $4.2 \mathrm{million}$ as at the 2006 census. The metropolis is estimated to have a population of 427,760 people. Made up of Hausa and Fulani majority and a minority of Zabarmawa and Tuareg and other non- indigenous settlers. The two major languages in the state is Hausa and Fulfulde which is spoken among the Fulani. The main occupation of the people is grain production and animal husbandry. Majority of the indigenous people practice agriculture. Crops produced include commercial crops like millet, sorghum, beans, rice and maize. Other occupations commonly practiced are dying, blacksmithing, weaving, carving, trading, and cobbling. Sokoto ranks second in livestock production in Nigeria. Modern Sokoto city is a major commercial center in leather crafts and agricultural products. Occupation of city inhabitants also include trading, commerce, with a reasonable proportion of the population working in private and public sectors [16].

Sociocultural characteristics is homogenous as majority of its indigenes and inhabitants are Muslims, therefore the doctrines of Islam provide the singular code of conduct and behavioral characteristics generally accepted across the State. Common practices are early marriage, polygamy, consanguity and multiple births.

\subsection{Study population}

The study was hospital based and involved all patients suspected with malaria attending specialist hospital and Maryam Abacha Women and Children hospitals, sokoto.

\subsection{Sample size determination}

The sample size was calculated by using the formula: $n=\frac{Z^{2} P \times q}{d^{2}}$. The calculated sample size was 297 . Therefore 297 febrile patients from specialist hospital and Maryam Abacha Women and children hospital were enrolled for this study.

\subsection{Sampling technique}

Simple random sampling technique was used for this study.

\subsection{Ethical approval}

The ethical approval was obtained from ethical and research committee of the two selected hospitals (Specialist hospital and Maryam abacha women and children hospital, sokoto). 


\subsection{Questionaire administration}

A semi structured interviewer administered questionnaire was used to obtain information on patients' sociodemographic characteristics and clinical factor such as names, age, sex, residence, occupation, educational status, duration of symptoms, medical history such as duration of fever, headache, chills, pains, vomiting, diarrhea, enlarged spleen, fatigue, presence of any underlying diseases.

\subsection{Sample collection}

Three millitres ( $3 \mathrm{mls}$ ) of venous blood was collected from each subject and transferred in to a clean EDTA container and mixed to avoid clot formation [17].

\subsection{Analytical procedure}

\subsubsection{Thick film making}

A drop of well mixed EDTA blood was placed on to the center of a clean grease free slide, it was spread in a circular pattern to make a thick smear measuring approximately $1 \mathrm{~cm}$ and 15 by $15 \mathrm{~mm}$ wide, in diameter using a spreader and was allowed to air dry [17].

\subsubsection{Thin film making}

A drop of blood was placed at one end of a well labelled clean grease free slide. A smooth edged spreader was placed at an angle of $45^{0}$ infront of the drop of blood. The drop of the blood was allowed to run along the edge of spreader. The spreader was pushed gently along the horizontal slide to make a thin smear with head body and tail. It was then allowed to air dry [17].

\subsubsection{Staining procedure for thick film}

The smear was flooded with $10 \%$ geimsa working solution, and it was then stained for 10 minutes on staining rack, after which, the slide was washed with buffered distilled water (PH 7.2). It was then allowed to air dry vertically and then the back of the slide was wiped with cotton wool [17].

\subsubsection{Staining procedure for thin film}

The air dried thin blood film was fixed in absolute methanol for two minutes. The smear was allowed to air dry and then flooded with $10 \%$ geimsa working solution. The slide was allowed to stain for 10 minutes, it was washed with distilled water and allowed to air dry [17].

\subsubsection{Microscopic examination}

The entire smears were first screened at low magnification $(\times 10$ and $\times 40$ objectives lens) to detect suitable fields with even distribution of WBC (10-20 WBC/field) and Red blood cells. Smears were then examined using $\times 100$ Objective. Atleast 100 high power fields were examined before a thick smear and thin smear was declared negative. Trophozoite and schizonts were counted per 200 or 500 leukocytes which were used to estimate the parasite density per microliter of blood in thick film examination [18].

\subsubsection{Parasite count and species identification}

The glass slide was placed on microscope with the label to the left. It was then examined by looking at the typical field with both parasite and white cell. After counting all parasites and white cells in one field, then moving to the next field and the counting procedure was repeated. The number of parasites were observed, the counting was stopped after examining 200 or 500 white cells. If 100 parasites or more were counted against 200 white cells, the counting was stopped and result was recorded as the number of parasites per 200 white cells. If after 200 WBCs have been counted, the number of parasites was 99 parasites or less than 99, counting was continued up to 500 WBCs, the result was recorded as number of parasite against 500 WBCs. The number of parasites relative to the number of WBCs was calculated and expressed as parasite per microliter of blood from mathematical formula below [19].

$$
\text { Parasite } / \mu \mathrm{l} \text { Blood }=\frac{\text { Number of parasite counted } \times 8000 \text { white cells } / \mu \mathrm{l}}{\text { Number of white cell counted. }}
$$

In thin film, morphological characteristics were used to identify Plasmodium species such as red blood cell enlargement, Stippling bodies, pigment, number of schizont, and trophozoites number. 


\subsection{Statistical analysis}

Data was presented in tables for descriptive purpose and analyzed using a statistical package for social science (SPSS) version 25. Pearson's correlation coefficient was used to establish association between parasite density and patients' gender and age. Independent sample t-test was used to obtain mean parasite density.

\section{Results}

Of the 297 subjects screened, 83were positive for malaria representing $28 \%$ prevalence and $214(72 \%)$ were tested negative. Plasmodium falciparum was responsible for all the cases of parasitaemia (table 1).

Table 1 Overall prevalence of malaria

\begin{tabular}{lcc}
\hline Malaria & Frequency (F) No. & Percentage (\%) \\
\hline Positive & 83 & 28 \\
Negative & 214 & 72 \\
Total & 297 & 100 \\
\hline \multicolumn{3}{c}{}
\end{tabular}

Based on gender, females had higher prevalence and parasite density of $57 \%$ and $690 \mu / 1$ respectively than males with prevalence of $43 \%$ and parasite density of $450 \mu / \mathrm{l}$. Positive correlation was observed between parasite density and gender of the subjects $(\mathrm{r}=0.103)$ while the $\mathrm{P}$ value was insignificant $(\mathrm{p}=0.76)$ as shown in table 2 .

Table 2 Prevalence of malaria and parasite density based on gender

\begin{tabular}{lllcl}
\hline Gender & $\begin{array}{l}\text { Positive } \\
\text { NO. (\%) }\end{array}$ & $\begin{array}{l}\text { Mean parasite } \\
\text { Density(parasite } / \boldsymbol{\mu l})\end{array}$ & r value & p value \\
\hline Male & $36(43)$ & 450 & 0.103 & 0.76 \\
Female & $47(57)$ & 690 & & \\
\hline \multicolumn{4}{r}{ r-positive correlation (significant), p-insignificant }
\end{tabular}

Prevalence of malaria based on age of the subjects showed that patients of $\leq 14$ years had higher prevalence of $43 \%$ followed by 15-24 years, 25-34 years, 35-44 years, with prevalence rates of $29 \%, 17 \%$ and $6 \%$ respectively, while patients of age range of 45-54 years had the lowest prevalence of 5\% (Table 3). There was positive correlation between parasite density and age group of the subjects $(r=0.020)$, while the $p$ value was insignificant $(p=0.731)$.

Table 3 Prevalence of malaria based on age

\begin{tabular}{llll}
\hline Age group (years) & Positive NO. (\%) & Negative NO. (\%) & Total NO. (\%) \\
\hline$\leq 14$ & $36(43)$ & $106(50)$ & 142 \\
$15-24$ & $24(29)$ & $56(26)$ & 80 \\
$25-34$ & $14(17)$ & $31(15)$ & 45 \\
$35-44$ & $5(6)$ & $9(4)$ & 14 \\
$45-54$ & $4(5)$ & $12(5)$ & 16 \\
Total (\%) & $83(28 \%)$ & $214(72 \%)$ & 297 \\
\hline \multicolumn{4}{l}{}
\end{tabular}

\section{Discussion}

Estimation of parasite density and identification of malaria parasite species is necessary for patients' management, especially when parasite resistance to available therapy is anticipated particularly in clinical trials or in drug efficacy studies. A convenient method of estimation using assumption constant WBC of $8000 \mathrm{cell} / \mu \mathrm{l}$ of peripheral blood by WHO is used for parasite density estimation, since most laboratories in malaria endemic countries in sub-Saharan Africa may not have the capacity to measure the WBC of each patient for parasite density estimation and microscopically knowledge of species identification is needed [19]. 
In this study, an overall prevalence of $28 \%$ was obtained and Plasmodium falciparum was responsible for all the cases of the infection recorded. A significant correlation coefficient was observed between the gender, and the P value was insignificant $(r=0.103$ and $p=0.76)$. Correlation coefficient of 0.020 and $p$ value of 0.731 was observed among age groups. The $P$ value was statistically insignificant but the $r$ value was significant. The findings of this study is in agreement with that of Abdullahi et al. [20], in Sokoto, which showed a prevalence of $27.29 \%$. The similarity of the results may be as a result of the same geographic regions where both studies were conducted. Higher prevalence rates of $32.5 \%$ and $41.6 \%$ than our finding were reported by Zama et al. [21] and Fana et al. [22] respectively. The differences may be as a result of immune status of the pregnant women used in their study. Plasmodium falciparum was responsible for all cases of parasitaemia. The study showed a higher prevalence of malaria and parasite density among female subjects $(57 \%$ and $690 \mu / \mathrm{l})$ than in Males $(43 \%$ and $450 \mu / \mathrm{l})$. The parasite density count is in contrast with that of Fana et al. [22], who reported a higher count of 800 parasite $/ \mu \mathrm{L}$ than our finding. This may be as a result of low immune status of the pregnant women recruited in their study. The prevalence with reference to age group of the subjects showed that patient's $\leq 14$ years had a highest prevalence of $43 \%$ and those of $15-24$ years showed a prevalence of $29 \%$, 25-34 years (17\%), 35-44 years (6\%) and 45-54 years had a lowest prevalence of 5\%. This showed that the density of the infection decreases with increase in age groups. The higher prevalence among age groups $\leq 14$ years seen in this study is in line with several studies [18, 20,21,23]. Malaria is common among children five years of age and less than 5 years, because there is slow acquisition of active immunity to malaria in those years, it is not surprising the situation is the same in Sokoto. Children born to immune mothers are protected against the disease during their first half year of life by maternal antibodies, as they grow they are exposed to the infection. But as they become older, after continued exposure from multiple infections with malaria parasites over time, they build up an acquired immunity and become relatively protected against disease and blood stage parasites hence lower prevalence of malaria among the older age groups of $45-54$ years.

\section{Conclusion}

Findings of this study reiterates the presence of Plasmodium falciparum in Sokoto metropolis. The overall infection rate is higher in females than in males, and age groups $\leq 14$ years are more infected. Parasite density count should be included in the diagnosis of malaria. Children should avoid playing in breeding sites of mosquitoes to minimize the risk of infection.

\section{Compliance with ethical standards}

\section{Acknowledgments}

We thank the entire staffs of department of medical microbiology, school of medical laboratory science, UDUS for their support towards the successful completion of this work.

\section{Disclosure of conflict of interest}

This article is not the object of any conflict of interest and has not been submitted to other journal for publication. Consequently, we authorize the journal to publish it.

\section{Statement of ethical approval}

Ethical approval was obtained from ethical and research committee of the two selected hospitals (Specialist hospital and Maryam abacha women and children hospital, sokoto).

\section{Statement of informed consent}

Informed consent was obtained from all individual participants included in the study.

\section{References}

[1] World health organization (2017). Geneva, WHO World Malaria Report.

[2] Fanello C, Onyamboko M and Lee SJ. (2017). Post-treatment hemolysis in African children with hyperparasitaemic falciparum malaria, a randomized comparison of artesunate and quinine. BMC Infect Dis., (17), 575. 
[3] Nkumama IN, Meara WP and Osier FH. (2017). Changes in malaria epidemiology in Africa and new challenges for elimination. Trends Parasitology, (33), 128-40.

[4] Breman JG, Alilio MS and Mills A. (2004). Conquering the intolerable burden of malaria: what's new, what's needed: a summary. American Journal of Tropical Medicine and Hygiene, 2(1), 95-101.

[5] Nadjm B and Behrens RH. (2012). Malaria: An update for physicians". Infectious Disease Clinics of North America, 26 (2), 243-59.

[6] Abdallah S and Karunamoorthi K. (2016). Malaria and blood transfusion: major issues of blood Safety in Malariaendemic countries and strategies for mitigating the risk of Plasmodium parasites. Parasitology Res, (115), 3547.

[7] World health organization. Basic malaria microscopy, Part I. Learner's guide. Second edition. Geneva. (2010).

[8] Mens PF, Schoone GJ, Kager PA and Schallig HP. (2006). "Detection and identification of Human Plasmodium species with real-time quantitative nucleic acid sequence-based amplification". Malaria Journal, 5 (80), 80.

[9] Collins WE. (2012). "Plasmodium knowlesi: A malaria parasite of monkeys and humans". Annual Review of Entomology, (57), 107-21.

[10] Snow RW, Sartorius B and Kyalo D. (2017). The prevalence of Plasmodium falciparum in sub-Saharan Africa since 1900. Nature, (550), 515-18.

[11] Singh B, Sung L and Matusop A. (2004). a large focus of naturally acquired Plasmodium knowlesi Infections in human beings. Lancet, (363), 1017-24.

[12] World health organization. (2014). World Malaria Report. Geneva, Switzerland, 32-4.

[13] Hisam S, Lanza M, Jiram AI, Ismail N and Rubio JM. (2014). First case of a Naturally acquired human infection with Plasmodium cynomolgi. Malaria Journal, (13), 68-70.

[14] Lyness R and David A. (1997). Diagnostic Medical parasitology 3rded. Washington. D.C: ASM press, 113-120.

[15] Fried M and Duffy PE. (2017). Malaria during pregnancy. Cold Spring Harbal Perspective Medicine, (7), a025551.

[16] MOI. In: Molineaux L and Gramaccia G. (2008). Research on the Epidemiology and Control of Malaria in the Sudan Savannah of West Africa. WHO Geneva, (311), 12.

[17] Cheesbrough M. (2005). District Laboratory Practice in Tropical Countries, part 1. University Press, Cambridge, 239-258.

[18] World health organization. (2015). National malaria slide bank standard operating procedures. Geneva.

[19] World health organization. (2014). World Malaria Report. Geneva, Switzerland, 32-4.

[20] Isah Z, Abdulrahaman Y, Augustine NO, Ishaku EY, Osaro E, Abdullah SM and Kufre EU. (2013). Prevalence of malaria parasitaemia and methaemoglobin levels among blood donors in Sokoto, Nigeria. Internal Medicine Inside, 1-4.

[21] Abdullahi K, Abubakar U, Adamu T, Daneji AI, Aliyu RU, Jiya N, Ibraheem MTO and Nata'ala SU. (2009). Malaria in Sokoto, North Western Nigeria. African Journal of Biotechnology, 8 (24), 7101-7105.

[22] Sani AF, Mohammed DAB, Sule AA, Umar AI and Nata'ala SU. (2015). Prevalence and risk factors associated with malaria infection among pregnant women in a semi-urban community of north-western Nigeria. Infectious Diseases of Poverty. 4, (24).

[23] Umar RA and Hassan SW. (2002). The Relationship between levels of parasitaemia and anaemia in children with malaria. Sahel Med. J., 5(1), 58-62.

\section{How to cite this article}

Bello M and Bello AT. (2019). Detection of Plasmodium species and parasite density among patients attending some selected hospitals in Sokoto Metropolis. GSC Biological and Pharmaceutical Sciences, 9(3), 01-07. 